\title{
Reliability of Frequency and Amplitude Decoding in Gene Regulation
}

\author{
Filipe Tostevin, Wiet de Ronde, and Pieter Rein ten Wolde \\ FOM Institute AMOLF, Science Park 104, 1098XE Amsterdam, Netherlands
}

(Received 4 May 2011; published 8 March 2012)

\begin{abstract}
In biochemical signaling, information is often encoded in oscillatory signals. However, the advantages of such a coding strategy over an amplitude-encoding scheme of constant signals remain unclear. Here we study the dynamics of a simple model gene promoter in response to oscillating and constant transcription factor signals. We find that in biologically relevant parameter regimes an oscillating input can produce a more constant protein level than a constant input. Our results suggest that oscillating signals may be used to minimize noise in gene regulation.
\end{abstract}

DOI: 10.1103/PhysRevLett.108.108104

PACS numbers: 87.18.Tt, 87.16.Yc, 87.18.Cf

Cells are constantly exposed to a range of environmental stimuli to which they must respond reliably. In recent years, it has become increasingly clear that cells use complex encoding strategies to represent information about the environment in the temporal dynamics of intracellular components [1]. In particular, oscillatory or pulsatile signals are commonly found in signaling and gene regulatory networks [2]. Perhaps the best known example is the phenomenon of calcium oscillations [3]. Oscillatory dynamics have also been observed at the level of gene regulation in nuclear localization of signaling proteins [4] and transcription factors [5-7] and in transcription factor expression [8]. Yet the advantages of such a coding strategy for signal transmission remain unclear.

A number of possible advantages for oscillatory signals have been suggested. Oscillatory signals minimize the prolonged exposure to high levels of calcium, which can be toxic for cells [9]. In systems with cooperativity [10] an oscillating signal effectively reduces the signal threshold for response activation. Pulsed signals also provide a way of controlling the relative expression of different genes [7]. However, these studies have ignored the impact of biochemical noise on the reliability of signal transmission. Encoding of signals in protein oscillations may play a direct role in ensuring accuracy in intracellular signaling.

Variability in the cellular response to an external signal will arise from the temporal pattern of network activation and from inevitable biochemical noise in the reactions making up the processing network, both of which will depend on the coding strategy employed. A reliable response requires minimization of such variability. Encoding of stimuli into oscillatory signals can reduce the impact of noise in the input signal and during signal propagation [11]. However, it remains unclear whether

Published by the American Physical Society under the terms of the Creative Commons Attribution 3.0 License. Further distribution of this work must maintain attribution to the author( $s)$ and the published article's title, journal citation, and DOI. oscillatory signals can also be decoded with a similar fidelity to constant signals-one might expect that the inherent variability of an oscillatory signal would inevitably lead to a more variable response. In this Letter we investigate whether oscillatory signals can be reliably decoded in a simple model of gene regulation. Surprisingly, we find that in biologically relevant parameter regimes an oscillating input can lead to a more constant output protein level than a constant input. This effect arises from differences in promoter-state fluctuations, which it has recently been shown can be a dominant source of noise in vivo [12].

We focus on the simple regulatory motif (Fig. 1) of a single gene promoter which can be in an active $\left(\mathrm{P}^{*}\right)$ or inactive $(P)$ state. The input to the system is the activity $s(t)$ of a transcription factor which enhances activation of the promoter. A protein $\mathrm{X}$, whose level $X(t)$ constitutes the network output, is transcribed from the active promoter; proteins also spontaneously decay. The system therefore consists of the reactions

$$
\mathrm{P} \underset{\lambda}{\stackrel{\kappa s(t)}{\rightleftharpoons}} \mathrm{P}^{*}, \quad \mathrm{P}^{*} \stackrel{\rho}{\rightarrow} \mathrm{P}^{*}+\mathrm{X}, \quad \mathrm{X} \stackrel{\mu}{\rightarrow} \varnothing .
$$

We will consider two forms for $s(t)$. In the first instance we take $s(t)=\alpha$ to be constant. In the second, $s(t)$ is oscillatory, reflecting the action of a periodic upstream signal or the inherently oscillatory dynamics of the transcription factor. Since transcription factor pulses often resemble distinct sharp peaks [5-7] we take $s(t)$ to be a binary switching process, with $s(t)=1$ for $n T \leq t<n T+\tau$

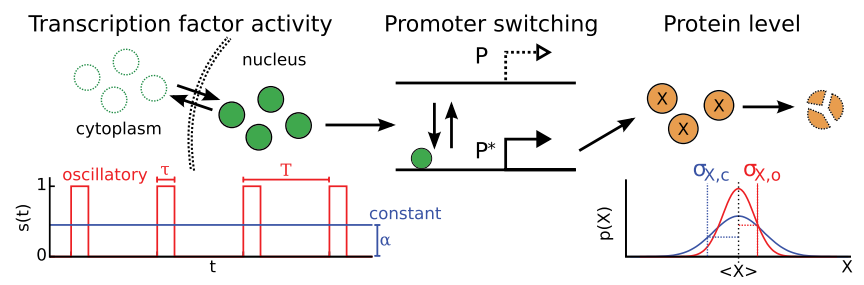

FIG. 1 (color online). A gene promoter driven by constant and oscillating signals, $s(t)$. We compare the variances of $X$ for the two input signals, $\sigma_{X, c}^{2}$ and $\sigma_{X, o}^{2}$, at the same mean level $\langle X\rangle$. 
$(n \in \mathbb{Z})$ and $s(t)=0$ otherwise (see Fig. 1). This form also has the advantage of making the dynamics analytically tractable. We characterize the system response in terms of the means $\langle X\rangle,\left\langle P^{*}\right\rangle$ and variances $\sigma_{X}^{2}, \sigma_{P^{*}}^{2}$; for the oscillating input we define the stationary mean $\langle X\rangle=$ $T^{-1} \int_{0}^{T} \mathbb{E}[X(t)] d t$, where $\mathbb{E}[]$ denotes averaging over network realizations with the same input, and the stationary variance $\sigma_{X}^{2}=T^{-1} \int_{0}^{T} \mathbb{E}\left[X(t)^{2}\right] d t-\langle X\rangle^{2}$. These are calculated from the chemical master equation [13], and verified by stochastic simulations [14].

When driven by a constant signal the promoter simply undergoes random switching with constant rates. Hence the probability of the promoter being active at any time is $\left\langle P^{*}\right\rangle_{c}=\alpha \kappa /(\alpha \kappa+\lambda)$. For an oscillating input the average activity,

$$
\left\langle P^{*}\right\rangle_{o}=\frac{\kappa}{\kappa+\lambda}\left[\frac{\tau}{T}+\frac{\kappa\left(1-e^{-(\kappa+\lambda) \tau}\right)\left(1-e^{-\lambda(T-\tau)}\right)}{\lambda T(\kappa+\lambda)\left(1-e^{-\kappa \tau-\lambda T}\right)}\right],
$$

contains a term from the expected promoter activity when $s=1$ multiplied by the fraction of time, $\tau / T$, for which $s(t)=1$, and a correction due to the fact that the promoter response time scales when $s(t)=1$ and $s(t)=0([\kappa+$ $\lambda]^{-1}$ and $\lambda^{-1}$, respectively) differ. Since promoter switching is a two-state process the variance in promoter activity is determined by the mean, $\sigma_{P^{*}}^{2}=\left\langle P^{*}\right\rangle\left(1-\left\langle P^{*}\right\rangle\right)$. The mean protein level also has the same form for both input signals, $\langle X\rangle=\rho\left\langle P^{*}\right\rangle / \mu$. However, differences in the timing of protein production will mean that the variance in the protein level differs between the two signals. For a constant signal the variance

$$
\sigma_{X, c}^{2}=\langle X\rangle_{c}\left[1+\frac{\rho \lambda}{(\alpha \kappa+\lambda)(\alpha \kappa+\lambda+\mu)}\right]
$$

consists of an intrinsic Poissonian term due to randomness in protein production, and an extrinsic contribution from fluctuations in the promoter state. The variance given an oscillatory input $\sigma_{X, o}^{2}$ can similarly be derived, but the full expression is unwieldy and thus not presented here. In the following we will compare $\sigma_{X, o}^{2}$ with $\sigma_{X, c}^{2}$ at the same mean response level, achieved by choosing the level of the constant signal $\alpha=\alpha(\tau, T, \kappa, \lambda)$ such that $\left\langle P^{*}\right\rangle_{c}=$ $\left\langle P^{*}\right\rangle_{o}=\left\langle P^{*}\right\rangle$, and hence also $\langle X\rangle_{c}=\langle X\rangle_{o}=\langle X\rangle$.

It is instructive to first consider cases in which the two input signals lead to similar distributions for $X$. First, if $\rho$, $\mu \rightarrow \infty$ with $X_{\infty}=\rho / \mu$ held constant, whenever the promoter is inactive the protein level is $X(t)=0$, while when the promoter is active the protein level will be Poissondistributed with mean $X_{\infty}$. The variance in $X$ is then given by $\sigma_{X, c}^{2}=\sigma_{X, o}^{2}=\langle X\rangle\left[1+X_{\infty}-\langle X\rangle\right]$. In this limit of fast protein dynamics the precise pattern of promoter switching does not affect the variance in protein expression. At the other extreme, if either promoter switching or protein production is slow compared to the oscillating input ( $\kappa$, $\lambda \ll T^{-1}$ or $\rho, \mu \ll T^{-1}$ ), the slow reactions effectively integrate over the temporal variation of the input. Since the network dynamics is too slow to reliably respond to the oscillating signal, the protein response is equivalent to that for the constant input.

Differences appear between the variances for the oscillatory and constant signals in the biologically most important regime of intermediate parameter values, where the promoter is able to respond to the oscillating input signal, protein production can react to switching of the promoter, and the protein lifetime is sufficiently long that patterns of promoter activity are important. Figures 2(a)-2(c) show that there exist regions in which either the constant or oscillating signal leads to smaller fluctuations $\sigma_{X}^{2}$ over large parameter ranges. Here we consider parameter ranges representative of eukaryotic cells, in which promoter switching occurs on a time scale of minutes to hours $[12,15]$ and protein lifetimes are in the range of a few to hundreds of hours [16]. The time scale of the input signal is chosen to be representative of NF- $\kappa \mathrm{B}$ oscillations [5]. However, we emphasize that out results are general and would apply equally to prokaryotic cells in an appropriate parameter regime.

To understand the noise properties for the two input signals we consider the network response in the frequency domain. The dynamics of $X(t)$ can be described by the Langevin equation

$$
\frac{d X}{d t}=\rho P^{*}(t)-\mu X(t)+\eta(t)
$$

where $\eta(t)$ represents Gaussian white noise with $\langle\eta(t)\rangle=0$ and $\left\langle\eta(t) \eta\left(t^{\prime}\right)\right\rangle=\left(\rho \mathbb{E}\left[P^{*}(t)\right]+\mu \mathbb{E}[X(t)]\right) \delta\left(t-t^{\prime}\right)$ [17]. Using the spectral addition rule [18] it is straightforward to calculate from Eq. (2) the power spectrum of fluctuations in $X(t), S_{X}(\omega)=\left[\rho^{2} S_{P}(\omega)+S_{\eta}(\omega)\right] /\left(\omega^{2}+\mu^{2}\right)$, in terms of the spectra of promoter fluctuations $S_{P}(\omega)$ and of intrinsic noise in the production and decay of $X, S_{\eta}(\omega)$. The variance $\sigma_{X}^{2}$ can be found by integrating $S_{X}(\omega)$ over all frequencies, and can therefore be written as the sum of intrinsic and extrinsic terms, $\sigma_{X}^{2}=\sigma_{\mathrm{ex}}^{2}+\sigma_{\text {in }}^{2}$ with

$$
\sigma_{\mathrm{ex}}^{2}=\int_{0}^{\infty} g^{2}(\omega) S_{P}(\omega) d \omega, \quad \sigma_{\mathrm{in}}^{2}=\int_{0}^{\infty} \frac{S_{\eta}(\omega)}{\omega^{2}+\mu^{2}} d \omega,
$$

where $g^{2}(\omega)=\rho^{2} /\left(\omega^{2}+\mu^{2}\right)$ is a signal-independent gain factor. The intrinsic noise $\sigma_{\mathrm{in}}^{2}=\langle X\rangle$ is also independent of the input signal. Any differences between $\sigma_{X, o}^{2}$ and $\sigma_{X, c}^{2}$ must, therefore, arise from differences in $S_{P}(\omega)$.

For a constant input, $S_{P, c}(\omega)=2 \sigma_{P^{*}}^{2} \tau_{P} /\left(\omega^{2} \tau_{P}^{2}+1\right)$ [Fig. 2(d), blue line] has a simple Lorentzian form due to random promoter switching, with $\tau_{P}=(\alpha \kappa+\lambda)^{-1}$ the switching correlation time. A general expression for $S_{P, o}(\omega)$ with an oscillating input is more difficult to calculate. However, simulations show [Fig. 2(d), red line] that there are two significant components. First, there are sharp peaks at frequencies corresponding to the signal period $\omega_{T}=2 \pi / T$ and multiples thereof, reflecting systematic changes in $\mathbb{E}\left[P^{*}(t)\right]$ due to the periodicity of $s(t)$. Second, 

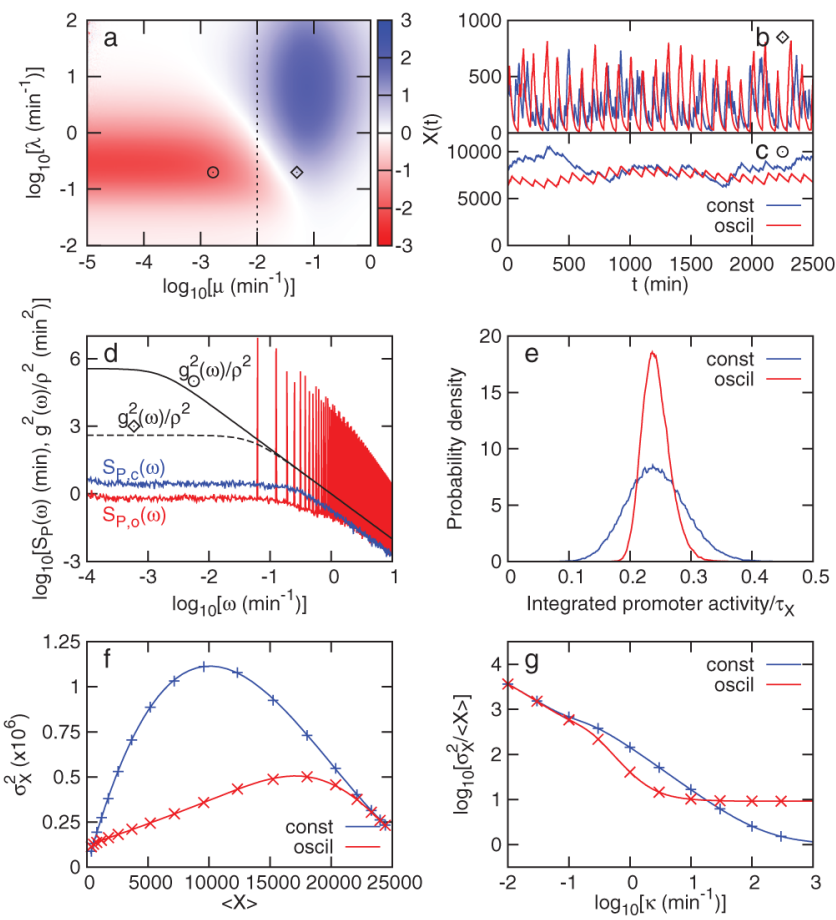

FIG. 2 (color online). (a) Relative noise for oscillating and constant signals, $\log _{2}\left(\sigma_{X, o}^{2} / \sigma_{X, c}^{2}\right)$, as the protein degradation rate $\mu$ and promoter deactivation rate $\lambda$ are varied. In red (blue) regions the oscillatory (constant) signal leads to lower noise. Other parameters are: $\tau=25 \mathrm{~min}, \quad T=100 \mathrm{~min}, \kappa=$ $1 \min ^{-1}, \rho=50 \mathrm{~min}^{-1}$. The dotted line indicates $T^{-1}$. (b),(c) Typical time-series of $X(t)$ for the parameter combinations denoted in (a) by $\diamond$ (b: $\lambda=0.2 \mathrm{~min}^{-1}, \mu=0.05 \mathrm{~min}^{-1}$ ) and $\bigcirc\left(\mathrm{c}: \lambda=0.2 \mathrm{~min}^{-1} \mu=0.00167 \mathrm{~min}^{-1}\right)$. Unless otherwise noted, parameters as in (c) are used in panels (d) $-(\mathrm{g})$. (d) The promoter power spectrum $S_{P}(\omega)$ and gain $g^{2}(\omega) / \rho^{2}=\left[\omega^{2}+\right.$ $\left.\mu^{2}\right]^{-1}$. For both inputs there is a noise background due to randomness in promoter switching. Peaks appear in $S_{P, o}(\omega)$ at $\omega=n \omega_{T}$ due to the periodicity of the oscillating input signal. (e) Distribution of the fraction of time the promoter is active in an interval $\tau_{X}$, calculated from stochastic simulations. The oscillatory signal leads to more reproducible promoter activity on the time scale $\tau_{X}$. (f) Variance against mean output level as $T$ is varied with $\tau$ held constant. The oscillating signal can achieve a lower variance over nearly the full range of output levels. In (f) and $(\mathrm{g})$, lines show exact analytic results, points show results of stochastic simulations. (g) Fano factor $\sigma_{X}^{2} /\langle X\rangle$ as $\kappa$ is varied with constant $K=\lambda / \kappa=0.2$. For slow switching $\kappa \tau \lesssim 1$ the two signals become equivalent. For extremely fast switching the constant signal minimizes $\sigma_{X}^{2}$. At intermediate switching rates, $0.1 \mathrm{~min}^{-1} \lesssim \kappa \lesssim 18 \mathrm{~min}^{-1}$, the oscillating signal allows for smaller $\sigma_{X}^{2}$.

$S_{P, o}(\omega)$ includes an approximately Lorentzian noise background associated with random (Poissonian) switching of the promoter when $s(t)=1$ and delays in deactivation when $s(t)=0$.

The protein lifetime $\tau_{X}=\mu^{-1}$ relative to the signal period $T$ is particularly important in determining which signal minimizes the output noise. The constant signal typically leads to smaller fluctuations when $\tau_{X}<T$ [see Fig. 2(a)]. In this regime, for the oscillatory signal $\sigma_{\mathrm{ex}}^{2}$ is dominated by contributions from the peaks of $S_{P, o}(\omega)$ appearing at $\omega=n \omega_{T}$, since $\mu \geqslant \omega_{T}$ and the gain $g^{2}(\omega)$ is large for frequencies $\omega<\mu$ [Fig. 2(d), dashed line]. Consequently, $X(t)$ features large production bursts when the signal is "on" with most proteins decaying before the next input pulse, while for a constant signal production and decay are more regularly distributed [see Fig. 2(b)].

For long protein lifetimes $\tau_{X}>T, \sigma_{X, o}^{2}$ is typically smaller than $\sigma_{X, c}^{2}$ [see Fig. 2(a)]. In this regime the impact of production bursts is reduced because proteins produced during many previous signal periods contribute to $X(t)$. Since $\mu<\omega_{T}$, the region $\omega \lesssim \mu$ where $g^{2}(\omega)$ is largest does not reach the first peak of $S_{P, o}(\omega)$ at $\omega_{T}$ [Fig. 2(d), solid black line]; hence $\sigma_{\mathrm{ex}}^{2}$ is dominated by promoter switching noise at low frequencies $\omega<\omega_{T}$, for which $S_{P, o}(\omega)<S_{P, c}(\omega)$. The large-amplitude changes of the oscillatory signal strongly bias the promoter to be active during a signal pulse and to be inactive between pulses, which in turn greatly reduces the probability of observing very long periods of promoter (in)activity. The elimination of such slow promoter fluctuations, which lead to the largest fluctuations in $X(t)$, means that on the time scale of the protein lifetime, promoter activity becomes more reproducible and the production of proteins becomes less variable when driven by an oscillatory signal [Fig. 2(e)]. Furthermore, Fig. 2(f) shows that in this regime $\sigma_{X, o}^{2} \leq$ $\sigma_{X, c}^{2}$ over nearly the full range of expression levels as the signal period $T$ is varied, indicating that this result does not require fine tuning of the reaction rates to the oscillation time scale.

Output noise $\sigma_{X}^{2}$ tends to decrease as promoter switching is made faster [Fig. 2(g)]: increasing the promoter switching rate reduces $S_{P}(\omega)$ at low frequencies, shifting power instead to high frequencies where $g^{2}(\omega) \sim \omega^{-2}$ is small. Interestingly, for extremely fast switching the constant signal is able to achieve a smaller variance. In this limit noise in promoter switching becomes negligible, and Eq. (1) reduces to

$$
\varnothing^{\rho^{\prime} s(t)} \stackrel{\mu}{\rightarrow} \varnothing .
$$

With a constant signal the effective production rate of $\mathrm{X}$ is $\rho_{c}^{\prime}=\rho\left\langle P^{*}\right\rangle / \alpha$ and $\sigma_{X, c}^{2}=\langle X\rangle=\sigma_{\text {in }}^{2} ; \sigma_{\text {ex }}^{2}$ [Eq. (3)] can be made arbitrarily small by shifting all promoter fluctuations to extremely high frequencies. With an oscillatory input the effective production rate becomes $\rho_{o}^{\prime}=\rho /(1+K)$, where $K=\lambda / \kappa$. The resulting variance,

$$
\sigma_{X, o}^{2}=\langle X\rangle\left[1+\frac{\rho_{o}^{\prime}}{\mu}\left(1-\frac{\tau}{T}-\frac{\left(1-e^{-\mu \tau}\right)\left(1-e^{-\mu(T-\tau)}\right)}{\mu \tau\left(1-e^{-\mu T}\right)}\right)\right],
$$

includes a (positive) extrinsic contribution. While the promoter switching noise background in $S_{P, o}(\omega)$ vanishes, as 
with a constant signal, the peaks due to the periodicity of $s(t)$ remain. Hence for an oscillatory input there will always be some overlap between $S_{P, o}(\omega)$ and $g^{2}(\omega)$, and a nonzero extrinsic noise $\sigma_{\mathrm{ex}}^{2}$. With the reaction rates representative of eukaryotic transcription used in Fig. 2(g), the crossover at which $\sigma_{X, o}^{2}=\sigma_{X, c}^{2}$ is $\kappa \approx 18 \mathrm{~min}^{-1}$. Experimentally determined rates of promoter activation, however, are typically $0.01-1 \mathrm{~min}^{-1}[12,15]$, which suggests that under biologically relevant conditions oscillatory signals can lead to more robust protein expression.

Thus far we have assumed that the signals $s(t)$ are deterministic. In reality, these signals will also be noisy as they are themselves generated by stochastic biochemical processes. An important question is whether oscillatory signals can still be decoded more reliably once noise in the input stimulus is taken into account.

We first consider the effect of transcription factor copynumber fluctuations around a constant mean by simulating $s(t)$ as a birth-death process. We find that $\sigma_{X}^{2}$ at fixed $\langle X\rangle$ increases monotonically as the variance or correlation time of $s(t)$ are increased. Therefore, without additional nonexponential temporal correlations in the input signal, noise is unable to reduce $\sigma_{X}^{2}$ below that achieved with $s(t)=\alpha$.

For an oscillatory input signal there are two principal types of noise: fluctuations in the amplitude and timing of signal pulses. First we perform simulations in which on each occasion that the oscillatory signal switches on the amplitude $s(t)=a$ is sampled from a log-normal distribution with mean $\bar{a}=1$ and width parameterized by $\xi^{2}=$ $\ln \left[\overline{a^{2}}\right]$. Figure 3(a) shows that $\sigma_{X}^{2}$ is largely unchanged until the noise in the pulse amplitude becomes large. Even with $\xi=1\left(\sigma_{a} / \bar{a} \approx 1.3\right)$ the output noise is typically smaller than for a constant, noiseless, input signal. Intuitively, in the regime where an oscillating signal leads to a smaller $\sigma_{X}^{2}, K \ll 1$; the promoter activity is driven to saturation during a signal pulse and hence amplitude fluctuations have little effect until there is a significant probability that $a \sim K$. The decoding of large-amplitude

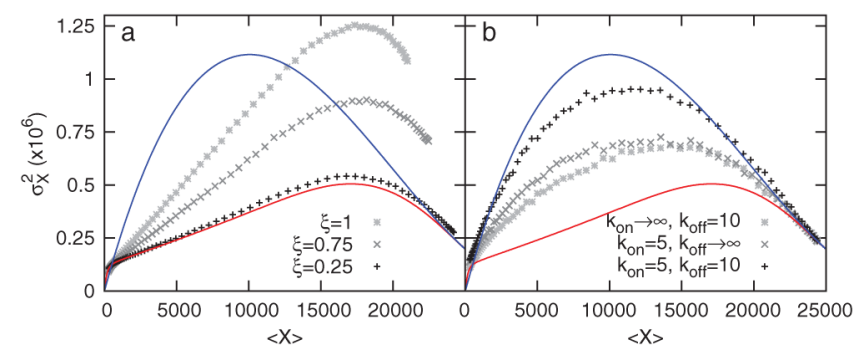

FIG. 3 (color online). Effects of noise in (a) oscillation amplitude, and (b) the duration of on and off periods. Solid lines show results for constant (blue) and deterministic oscillatory (red) stimuli. Even with significant variability in amplitude or timing, the oscillating signal leads to a smaller $\sigma_{X}^{2}$ than a constant signal. Parameters are as in Fig. 2(f). oscillatory signals is therefore highly robust to noise in the oscillation amplitude.

We next investigate by simulations the effect of noise in the duration of signal pulses and interpulse intervals. The duration of each on period is chosen independently from a gamma distribution with mean $\tau$ and shape parameter $k_{\mathrm{on}}$. The resulting variance in on durations is $\sigma_{t_{\mathrm{on}}}^{2}=\tau^{2} / k_{\mathrm{on}}$. "Off" periods are similarly sampled from a distribution with mean $T-\tau$ and parameter $k_{\text {off }}$. Figure 3(b) shows that the noisy oscillation can lead to protein level fluctuations which are similar to or smaller than $\sigma_{X, c}^{2}$ even when variability in signal timing is significant $\left(k_{\text {on }}=5\right.$ gives $\sigma_{t_{\mathrm{on}}} / \tau \approx 0.45$ ). Experimentally-observed fluctuations in oscillation periods or peak widths vary between different systems but can be 20\%-30\% [4,19], suggesting that in vivo oscillations can be sufficiently precise as to reduce output noise compared to a constant-amplitude signal.

The simple model of gene expression considered here neglects mRNA dynamics and processing. Such processes can affect the propagation of promoter-state fluctuations in two ways. First, mRNA dynamics will integrate over promoter fluctuations on time scales shorter than the mRNA lifetime (typically tens to hundreds of minutes in eukaryotic cells $[16,20])$. However, differences between $\sigma_{X, c}^{2}$ and $\sigma_{X, o}^{2}$ are primarily due to promoter-state fluctuations on time scales comparable to or longer than the slowest time scale of variations in the protein level, which is typically determined by the protein lifetime (ten to a hundred hours [16]). Promoter fluctuations on these long time scales can not be filtered by the mRNA dynamics, and hence even with mRNA dynamics taken into account an oscillatory input will lead to more robust expression since an oscillatory signal suppresses promoter fluctuations on time scales longer than the signal period [see Fig. 2(d)]. Second, a (random) delay between transcription initiation and protein synthesis will be introduced. However, such delays will only significantly affect the number of proteins produced on the time scale $\tau_{X}$ if the width of the delay distribution itself becomes comparable to the protein lifetime, which seems unrealistic. Hence we conclude that mRNA dynamics will have little effect on our results.

It is believed that biochemical networks employ frequency-encoding schemes in which stimuli are represented in the frequency of oscillations of signaling molecules $[3,7]$. Our results suggest that frequency encoding may allow for more reliable signaling than amplitudeencoding schemes because oscillatory signals can be decoded more reliably.

We thank Andrew Mugler for comments on the manuscript. This work is part of the research program of the "Stichting voor Fundamenteel Onderzoek der Materie (FOM)", which is financially supported by the "Nederlandse organisatie voor Wetenschappelijk Onderzoek (NWO)". 
[1] M. Behar and A. Hoffmann, Curr. Opin. Genet. Dev. 20, 684 (2010).

[2] P. Paszek, D. A. Jackson, and M. R. H. White. Curr. Opin. Genet. Dev. 20, 670 (2010).

[3] M. J. Berridge and A. Galione, The FASEB J. 2, 3074 (1988).

[4] H. Shankaran et al., Mol. Syst. Biol. 5, 332 (2009).

[5] D. E. Nelson et al., Science 306, 704 (2004).

[6] M. Jacquet et al., J. Cell Biol. 161, 497 (2003).

[7] L. Cai, C. K. Dalal, and M. B. Elowitz, Nature (London) 455, 485 (2008).

[8] G. Lahav et al., Nat. Genet. 36, 147 (2004).

[9] B.F. Trump and I. K. Berezesky, The FASEB J. 9, 219 (1995).

[10] D. Gall, E. Baus, and G. Dupont, J. Theor. Biol. 207, 445 (2000).
[11] P. E. Rapp, A. I. Mees, and C. T. Sparrow, J. Theor. Biol. 90, 531 (1981).

[12] D. M. Suter et al., Science 332, 472 (2011).

[13] N. G. van Kampen, Stochastic Processes in Physics and Chemistry (North Holland, Amsterdam, 1992).

[14] D. T. Gillespie, J. Comput. Phys. 22, 403 (1976).

[15] J. R. Chubb et al., Curr. Biol. 16, 1018 (2006).

[16] B. Schwanhäusser et al., Nature (London) 473, 337 (2011).

[17] P. B. Warren, S. Tănase-Nicola, and P. R. ten Wolde, J. Chem. Phys. 125, 144904 (2006).

[18] S. Tănase-Nicola, P. B. Warren, and P. R. ten Wolde, Phys. Rev. Lett. 97, 068102 (2006).

[19] N. Geva-Zatorsky et al., Mol. Syst. Biol. 2, 0033 (2006).

[20] M. Rabani et al., Nat. Biotechnol. 29, 436 (2011). 